\title{
ERK1/2-Akt1 crosstalk regulates arteriogenesis in mice and zebrafish
}

\author{
Bin Ren, ${ }^{1}$ Yong Deng, ${ }^{2}$ Arpita Mukhopadhyay, ${ }^{3}$ Anthony A. Lanahan, ${ }^{2}$ Zhen W. Zhuang, ${ }^{2}$ \\ Karen L. Moodie, ${ }^{4}$ Mary Jo Mulligan-Kehoe, ${ }^{4}$ Tatiana V. Byzova, ${ }^{5}$ \\ Randall T. Peterson, ${ }^{3}$ and Michael Simons ${ }^{2,6}$
}

\begin{abstract}
1Department of Cell Biology, Cleveland Clinic Foundation, Ohio. 2Section of Cardiovascular Medicine, Department of Internal Medicine, Yale University School of Medicine, New Haven, Connecticut. ${ }^{3}$ Cardiovascular Research Center, Massachusetts General Hospital, Harvard Medical School, Charlestown. ${ }^{4}$ Department of Medicine, Dartmouth Medical School, Lebanon, New Hampshire. 5Department of Molecular Cardiology, Cleveland Clinic Foundation. ${ }^{6}$ Department of Cell Biology, Yale University School of Medicine.
\end{abstract}

\begin{abstract}
Arterial morphogenesis is an important and poorly understood process. In particular, the signaling events controlling arterial formation have not been established. We evaluated whether alterations in the balance between ERK1/2 and PI3K signaling pathways could stimulate arterial formation in the setting of defective arterial morphogenesis in mice and zebrafish. Increased ERK1/2 activity in mouse ECs with reduced VEGF responsiveness was achieved in vitro and in vivo by downregulating PI3K activity, suppressing Akt1 but not Akt2 expression, or introducing a constitutively active ERK1/2 construct. Such restoration of ERK1/2 activation was sufficient to restore impaired arterial development and branching morphogenesis in synectin-deficient mice and synectin-knockdown zebrafish. The same approach effectively stimulated arterial growth in adult mice, restoring arteriogenesis in mice lacking synectin and in atherosclerotic mice lacking both LDL-R and ApoB48. We therefore conclude that PI3K-ERK1/2 crosstalk plays a key role in the regulation of arterial growth and that the augmentation of ERK signaling via suppression of the PI3K signaling pathway can effectively stimulate arteriogenesis.
\end{abstract}

\section{Introduction}

Effective stimulation of arteriogenesis has long been an important therapeutic aim in clinical medicine. Attempts at stimulating it using various growth factors have failed largely because of impaired signaling of VEGF (and likely other growth factors) in vascular tissues in patients with advanced vascular diseases (1) caused by a combination of atherosclerosis (2), diabetes (3), age, and other factors (4). Therefore, we have sought to establish a signaling pathway responsible for arterial growth that could be activated downstream of impaired growth factor signaling.

Arterial morphogenesis is a complex and poorly understood process that involves a number of external clues that direct acquisition of an arterial identity by a newly formed vascular structure, followed by its maturation. To date, only a few such clues have been identified, including the sonic hedgehog-VEGF-A-Notch and TGF- $\beta$ cascades (5) that appear to transmit an arterial specification signal, and PDGF-BB signaling that is critical to maturation of a newly formed vessel $(6,7)$. Although our understanding of arteriogenic clues is far from complete, even less is known about intracellular endothelial signaling events that result in the appearance of an arterial phenotype.

Several recent studies have pointed to a potential role of ERK in this process. High levels of phosphorylated ERK1/2 (P-ERK1/2) are found in growing collateral arteries (8). Shear stress, a major factor thought to trigger arteriogenesis (4), is capable of inducing ERK1/2 activation $(9,10)$. Furthermore, ERK1/2 phosphorylation is profoundly reduced in the posterior trunk vasculature of gridlock zebrafish mutants that demonstrate a failure of development of the distal arterial tree (11).

The gridlock phenotype is a consequence of a mutation in the gridlock gene $(g r l)$, which codes for a helix-loop-helix protein of the hairy and enhancer of split family of transcriptional repres-

Conflict of interest: The authors have declared that no conflict of interest exists. Citation for this article: J Clin Invest. 2010;120(4):1217-1228. doi:10.1172/JCI39837. sors. The mutant fish have incompletely formed lateral aortae and lack circulation in the posterior trunk and tail. The latter occurs via localized obstruction of the caudal blood flow at the base of the dorsal aorta while cranial circulation remains normal $(12,13)$. Activation of ERK1/2 in these mutants by means of partial inhibition of PI3K activity led to a virtually complete recovery of the distal arterial circulation (14). Thus, although $g r l$ does not represent a true failure of arterial development, it nevertheless points to an important role of ERK activation in this process.

We have recently demonstrated that deletion or knockdown of the PDZ scaffold protein synectin (also known as GIPC1) results in marked and selective impairment of arteriogenesis in both mice and zebrafish, with adult animals demonstrating poorly developed and hypobranched arterial networks in many organs $(15,16)$. Synectin has been isolated as a binding partner of a number of plasma cell membrane receptors, including syndecan-4 (17), neuropilin-1 (18), TrkA (19), and type III TGF- $\beta$ receptor (20), among others. Although its precise functional role has not been established, it is involved in regulation of a number of cellular process, such as migration, adhesion, and endocytosis (21-24), and synectin-deficient arterial ECs (AECs) demonstrate impaired migration and proliferation in response to VEGF (15). Thus, mice deficient for synectin present an interesting model to examine arterial morphogenic signaling. Atherosclerosis is another well-established factor that impairs growth factor signaling. In particular, mice lacking both LDL-R and ApoB48 ( $\left(\mathrm{dll} \mathrm{r}^{-}\right.$ApoB48-modified mice) that were fed a high-fat diet demonstrated impaired arteriogenesis after common femoral artery ligation and reduced responsiveness to angiogenic growth factors (2).

In this study, we investigated whether abnormal activation of ERK signaling may be responsible for impaired arteriogenesis and whether restoration of ERK signaling would restore effective arterial morphogenesis in these models. To achieve this goal, we used a strategy of partial PI3K inhibition that was previously shown to be effective in 
A

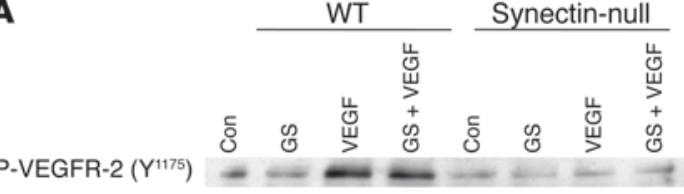

T-VEGFR-2

B

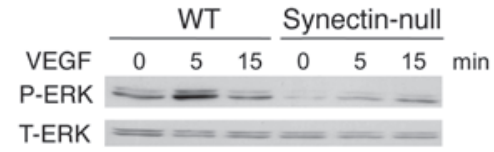

C $\quad$ VEgF $\begin{array}{llllllllll}0 & 2 & 5 & 10 & 0 & 2 & 5 & 10 & \mathrm{~min}\end{array}$

P-Akt $^{\mathrm{S} 73}$ - - - - - -

T-Akt

Synectin

D

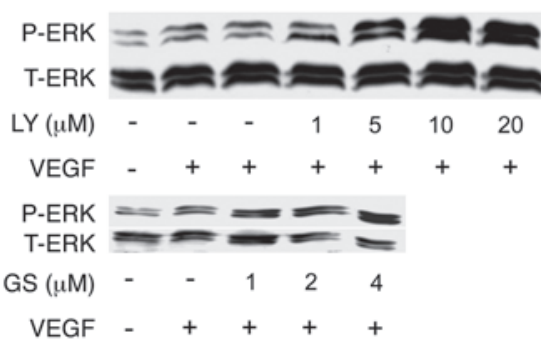

E

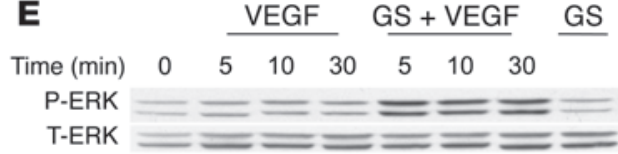

$\mathbf{F}$
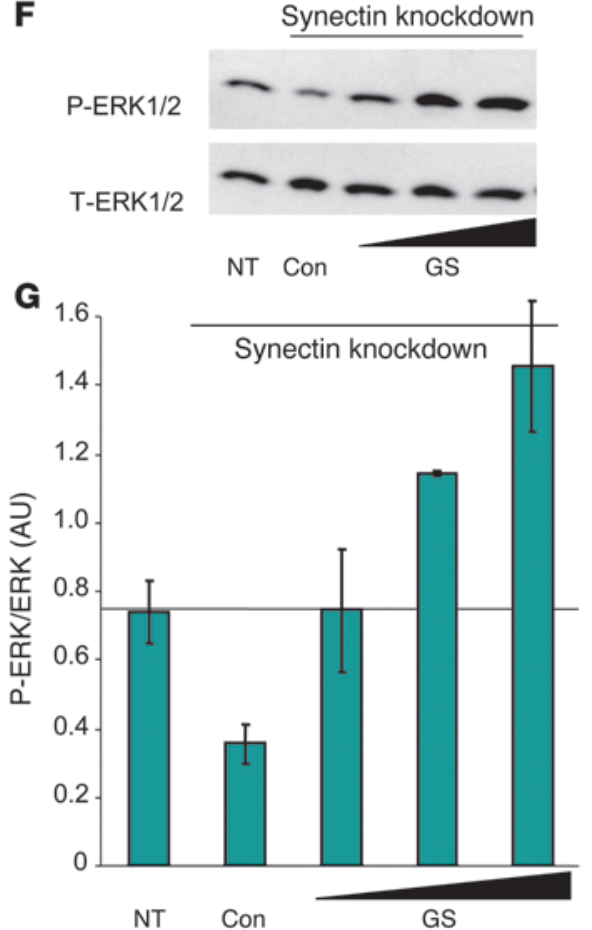

Figure 1

PI3K suppression activates ERK signaling in synectin-deficient AECs and in zebrafish. (A-E) Western blots of AECs from WT and synectin-null mice after treatment with VEGF-A ${ }_{165}$ in vitro. (A) Activation of the VEGFR2 $\mathrm{Y}^{1175}$ site by VEGF-A. Data shown are for 10 minutes after VEGF stimulation. T-, total. (B) Time course of ERK activation after VEGF-A 165 treatment. Note reduced ERK activation in synectin-deficient AECs. (C) Time course of Akt activation after VEGF-A 165 treatment. Note reduced AKT activation in synectin-deficient AECs. (D) Restoration of ERK activation by PI3K inhibition. Synectin-deficient AECs were stimulated with VEGF-A in the presence of increasing concentrations of LY294002 or GS4898, and ERK activation was tested 10 minutes later. Note dose-dependent increase in ERK activation. (E) Time course of ERK activation in synectin-deficient AECs by PI3K inhibition. Note long-term restoration of activity. (F and $\mathbf{G}$ ) Analysis of P-ERK1/2 and total ERK1/2 expression in zebrafish embryos. At 1-ss, embryos were injected with $12 \mathrm{ng}$ anti-synectin MOs or left untreated (NT). At 8-ss, injected embryos were treated with 20,50 , or $100 \mu \mathrm{M}$ GS4898 or DMSO as a control. (F) Representative Western blot. (G) Quantitative analysis of P-ERK/T-ERK ratio.

restoring the grl phenotype (14). The premise of this approach is the existence of a putative negative Akt-Raf1 feedback pathway, demonstrated in cell culture but not in vivo, that limits ERK activation (25).

We demonstrated that ERK1/2 activation was markedly reduced in synectin-deficient AECs in response to VEGF-A. An increase in ERK1/2 activity, either by partial inhibition of PI3K activity or by direct introduction of a constitutively active MEK-ERK construct, increased the ability of synectin-deficient AECs to migrate and to form branching vascular structures in vitro and restored arterial morphogenesis in synectin-knockdown zebrafish. Furthermore, the same manipulations were effective in restoring arteriogenesis in adult synectin-null mice and in $\mathrm{Ldlr}^{--}$ApoB48-modified mice maintained on a high-fat diet.

\section{Results}

Partial inbibition of PI3K signaling restores VEGF-dependent activation of ERK1/2 in synectin-deficient AECs. Synectin-deficient AECs were previously reported to demonstrate decreased responsiveness to VEGF (15). To examine the reason for this decreased respon- siveness and to elucidate the role of ERK activation in arterial morphogenesis, we first examined VEGFR2 activation on the site $\left(\mathrm{Y}^{1175}\right)$ known to control both PLC $\gamma$-ERK1/2 and PI3K-Akt pathways. Blotting with an anti-Y ${ }^{1175} \mathrm{Ab}$ demonstrated reduced phosphorylation in synectin-deficient compared with synectinsufficient WT AECs (Figure 1A). In agreement with reduced phosphorylation on this site, Western blotting with an anti-P-ERK1/2 $\mathrm{Ab}$ demonstrated markedly reduced ERK activation in synectin-deficient compared with WT AECs and a less pronounced decrease in Akt activation (Figure 1, B and C).

To test the hypothesis that inhibition of PI3K activity would stimulate ERK1/2 activity, synectin-deficient AECs were exposed to VEGF in the absence and presence of 2 different PI3K inhibitors: LY294002 and GS4898. Treatment with either LY294002 or GS4898 in conjunction with VEGF resulted in robust, dose-dependent ERK1/2 activation that was sustained for an extended period of time (Figure 1, D and E). Furthermore, GS4898 by itself, in the absence of VEGF, had no effect on ERK1/2 activation (Figure 1E). As expected with a PI3K inhibitor, Akt activation after GS4898 
A

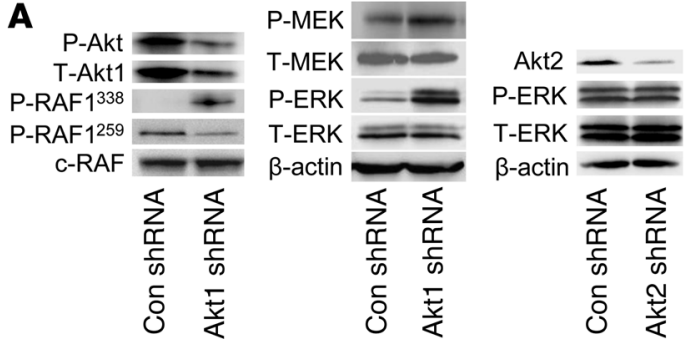

B

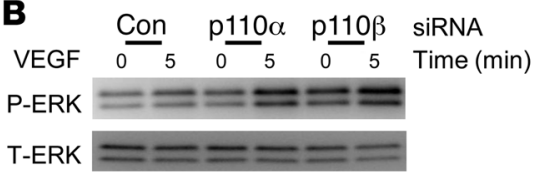

C

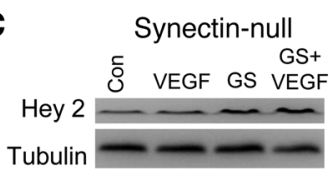

D Hey2 Liver

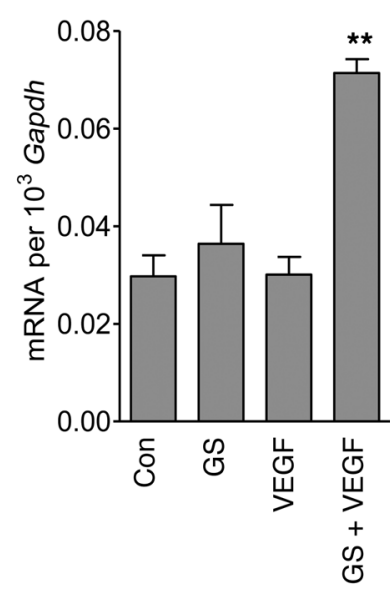

Ephrin B2 Liver

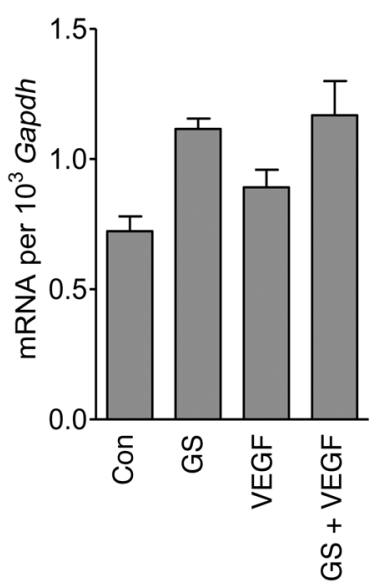

Ephrin B2 Heart

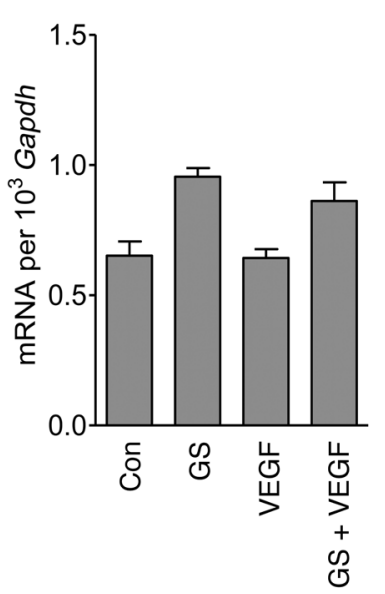

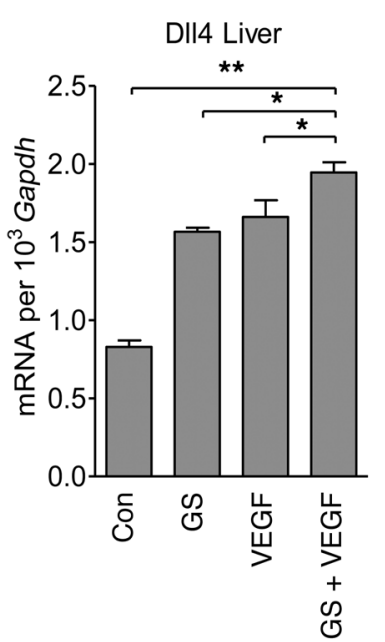

Figure 2

PI3K suppression restores arterial marker expression in synectin-deficient AECs. (A) Akt1 and Akt2 knockdown in synectin-deficient AECs. Synectin-deficient AECs were treated with Akt1- or Akt2-specific shRNA or a control shRNA. 24 hours later, cells were subjected to Western

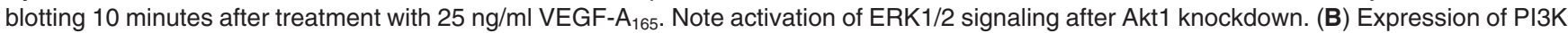
subunits $\mathrm{p} 110 \alpha$ and $\mathrm{p} 110 \beta$ in synectin-deficient AECs was reduced using specific siRNA or a nonspecific sequence as a control. 24 hours later, the cells were treated with VEGF, and ERK activation was determined at baseline (0 minutes) or at 5 minutes. Note increased ERK1/2 activation in cells treated with either $\mathrm{p} 110 \alpha$ or $\mathrm{p} 110 \beta$ siRNA. (C) Synectin-null $(n=6)$ and $L d l r^{-1}$ ApoB48-modified mice $(n=12)$ were treated for 3 days with GS4898 via an implanted Alzet minipump followed by VEGF-A injection. Shown are Western blots for hey2 and tubulin (loading control) in mice exposed to VEDGF alone, GS4898 alone, or a combination of both. Note decreased hey2 activation following a combination of VEGF and GS4898. (D) Quantitative PCR analysis of arterial marker expression. Synectin-null mice $(n=6)$ were treated as described above. Quantitative PCR was used to determine Hey2, ephrin B2, and DLL4 expression. ${ }^{*} P<0.05,{ }^{* *} P<0.01$ vs. control.

treatment was suppressed in a dose-dependent manner and for an extended period of time, as was activation of various Akt target genes (Supplemental Figure 1, A-C; supplemental material available online with this article; doi:10.1172/JCI39837DS1). Importantly, while restoring ERK1/2 activation, neither GS4898 nor LY294002 had any effect on VEGFR2 $\mathrm{Y}^{1175}$ phosphorylation (Figure 1A and data not shown). Thus, partial inhibition of PI3K/Akt signaling in the setting of VEGF stimulation resulted in activation of ERK1/2 that was independent of VEGFR2 activation.

We next evaluated the link between ERK1/2 activation and PI3K inhibition in vivo. Western blotting of zebrafish embryos with an anti-P-ERK1/2 Ab carried out on embryos at the 20 -somite stage (20-ss) subjected to synectin knockdown sufficient to disrupt arterial morphogenesis revealed a marked reduction in total P-ERK1/2 levels (Figure 1, F and G). After treatment with GS4898, we observed a dose-dependent increase in P-ERK1/2 levels that, at high doses, exceeded P-ERK1/2 levels in control embryos (Figure 1, F and G).
Akt1 controls ERK1/2 activation in AECs. Because Akt has previously been reported to inhibit ERK1/2 activation by phosphorylating Raf1, we tested the possibility that PI3K inhibition activates ERK1/2 by removing the Akt-inhibitory signal. To demonstrate this mechanism of action and to determine which Akt isoform is involved in this process, we decreased Akt 1 and Akt 2 expression in synectindeficient AECs using siRNA. The reduction in Akt 1 expression resulted in increased phosphorylation of $S^{338}$ in Raf- 1 and decreased phosphorylation of $\mathrm{S}^{259}$, a pattern of phosphorylation observed in active Raf-1 (Figure 2A). In agreement with this evidence of Raf-1 activation, there was an increase in P-MEK and P-ERK1/2 levels. In contrast, Akt2 knockdown had no effect on ERK1/2 activation.

Recent studies have suggested that the $\mathrm{p} 110 \alpha$ subunit of PI3K regulates angiogenesis and $\mathrm{EC}$ migration (26), whereas the $\mathrm{p} 110 \beta$ subunit regulates cell proliferation in a poorly understood, nonkinase-dependent manner (27). To determine which of these isoforms controls PI3K-Akt-dependent inhibition of ERK signaling, we knocked down both $\mathrm{p} 110 \alpha$ and $\mathrm{p} 110 \beta$ in synectin-defi- 
A

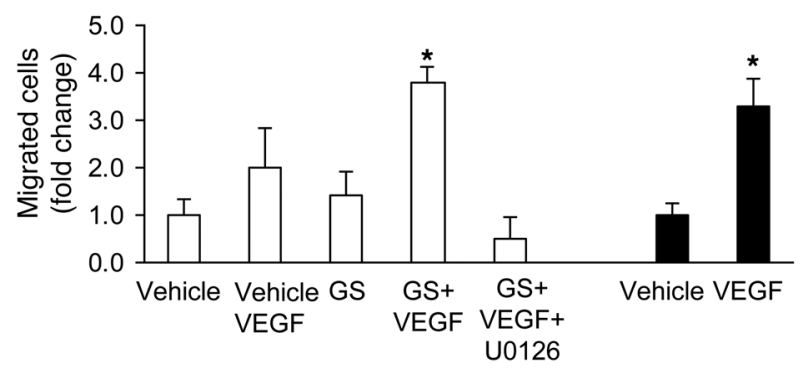

Synectin-null
B

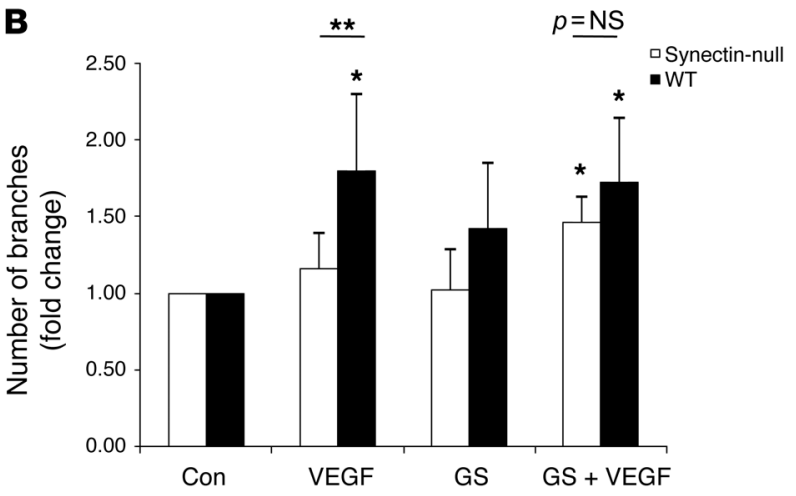

C

Synectin-null

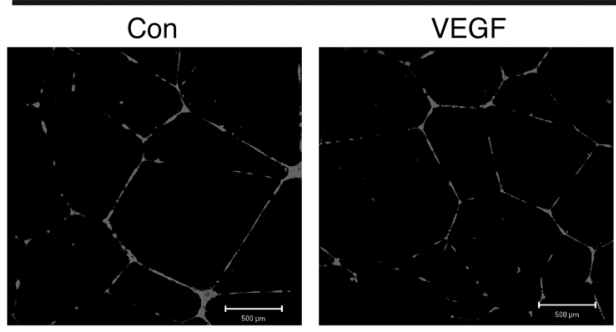

WT
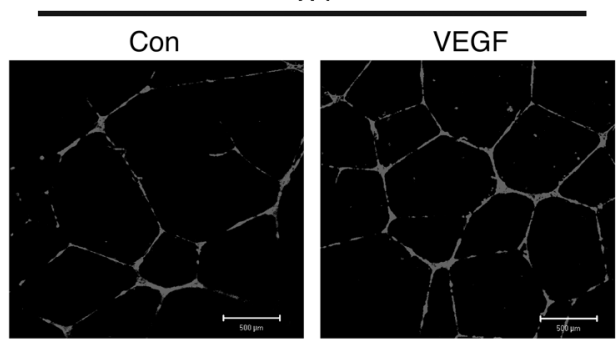

D

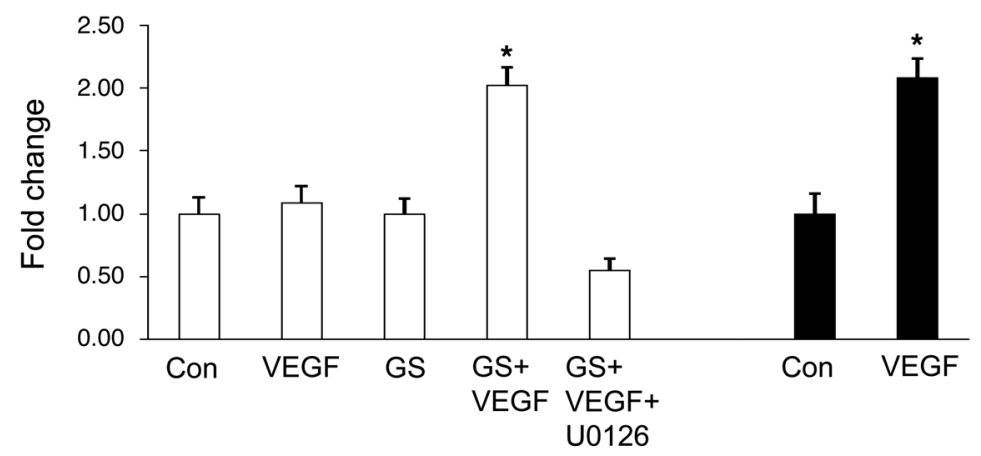

Figure 3

Functional effects of partial PI3K inhibition in synectin-deficient AECs. (A) Cell migration was assessed in the modified Boyden chamber assay.

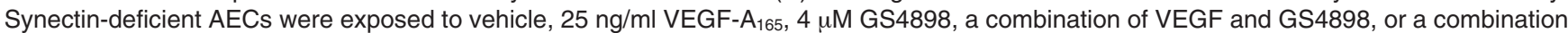
of GS4898 and VEGF in the presence of $10 \mu \mathrm{M}$ U0126. WT AECs were treated with either vehicle or VEGF-A 165 . The extent of migration is expressed as a ratio relative to vehicle-treated synectin-deficient AECs. Note full restoration of migration of synectin-deficient AECs exposed to GS4898 in the presence of VEGF. (B) 3D collagen tube branching assay. Synectin-deficient and WT AECs were placed in 3D collagen in the presence of $25 \mathrm{ng} / \mathrm{ml}$ VEGF, $4 \mu \mathrm{M}$ GS4898, or both. The extent of branching was assessed 24 hours later and expressed as fold change relative to untreated control synectin-deficient AECs. Note restoration of branching in synectin-deficient AECs by GS4898 treatment. (C and D) In vitro Matrigel. The extent of cords branching was assessed in synectin-deficient AECs placed on growth factor-depleted Matrigel and exposed to $25 \mathrm{ng} / \mathrm{ml}$ VEGF-A ${ }_{165}, 4 \mathrm{uM}$ GS4898, a combination of VEGF and GS4898, or a combination of GS4898 and VEGF in the presence of $10 \mathrm{uM}$ U0126. WT AECs exposed to VEGF were used as a control. All results are expressed relative to untreated control synectin-deficient AECs. Note restoration of branching in synectin-deficient AECs by GS4898 treatment. ${ }^{\star} P<0.05,{ }^{\star \star} P<0.001$ vs. control. Scale bars: $500 \mu \mathrm{m}$. 
A

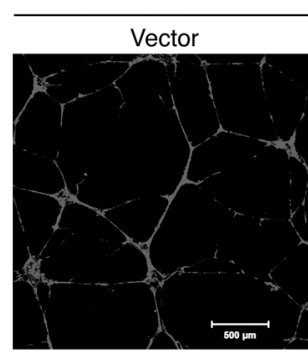

$\mathrm{ME}+\mathrm{VEGF}$
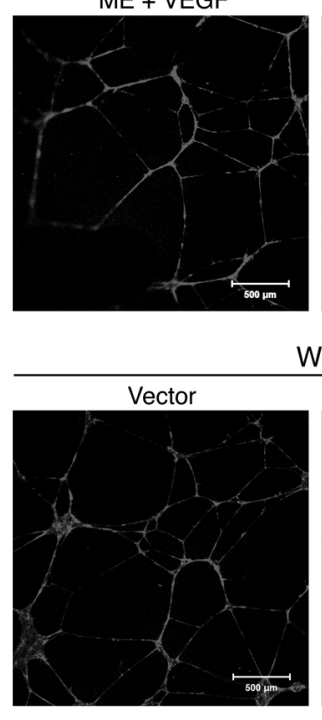

WT

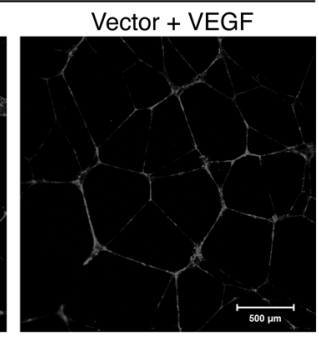

B

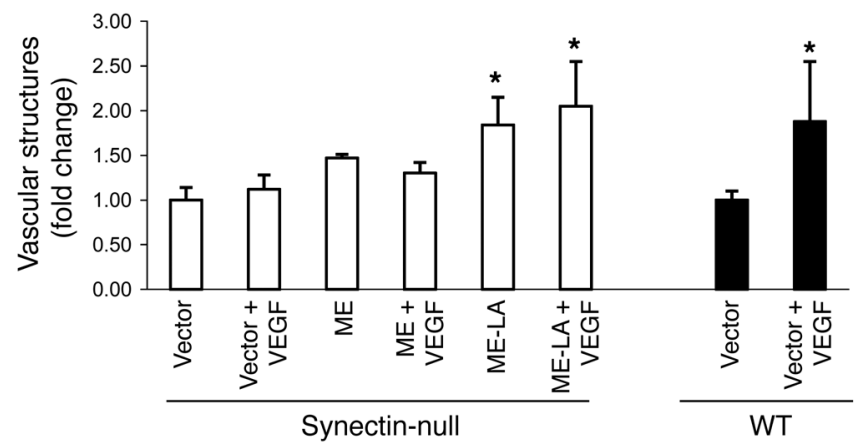

C

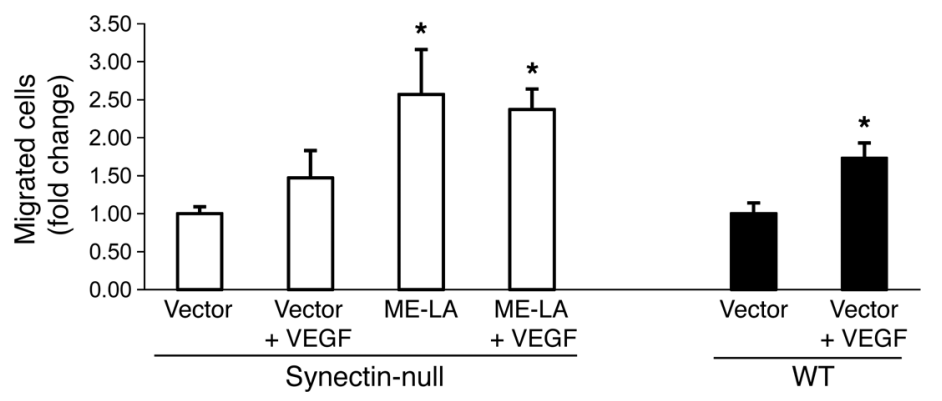

Figure 4

ERK activation restores functional defects in synectindeficient AECs. (A and B) Synectin-deficient AECs were transduced with empty Ad vector, Ad-ME, or Ad-ME-LA. The transduced cells were then plated in growth factordepleted Matrigel and exposed to $25 \mathrm{ng} / \mathrm{ml}$ VEGF-A 165 as indicated. The extent of cord branching, assessed 24 hours later as described in Figure 3, is expressed as fold change relative to untreated control synectin-deficient AECs. Note restoration of branching in synectin-deficient AECs expressing ME-LA construct. Scale bars: $500 \mu \mathrm{m}$. (C) Cell migration in modified Boyden chamber. Synectin-deficient or WT AECs, treated as above, were placed in the chamber, and the extent of migration in response to VEGF was assessed. Values are expressed as fold change relative to that of vehicle-treated synectin-deficient AECs. Note restoration of migration by the ME-LA construct. ${ }^{*} P<0.05$ vs. vector control.

reduced arterial marker expression (15). To determine whether restoration of ERK activation by partial inhibition of PI3K signaling affects this, synectin-null and Ldlr $/$ ApoB48-modified mice were treated for 3 days with daily doses of GS4898 and then given a single injection of VEGF-A. Western blotting of various tissues demonstrated increased expression of Hey 2 after this treatment, whereas, as expected, VEGF alone had little effect (Figure 2C). To confirm and expand these findings, we used quantitative PCR to measure Hey2, ephrin B2, and DLL4 expression in the liver and heart. This confirmed a major increase in Hey 2 expression after treatment with the combination of GS4898 and VEGF (Figure 2D). Treatment with GS4898 alone was sufficient to induce significant increases in ephrin B2 and DLL4 expression, although the latter was further augmented by a combination treatment.

ERK1/2 activation via PI3K suppression restores function of synectin-deficient $A E C$ s in vitro. To test the functional impact of restoration of ERK1/2 activation by means of partial PI3K activity suppression, we first tested the ability of synectin-deficient AECs to migrate and form branching structures in response to VEGF in vitro. As expected, synectindeficient AECs demonstrated impaired VEGF-induced migration. Exposure to GS4898 by itself had no effect, whereas a combination of VEGF and GS4898 restored cell migration to the level observed in WT cells in response to VEGF alone (Figure 3A). Inhibition of ERK activation by the MEK inhibitor U0126 fully blocked GS4898/VEGFdriven restoration of synectin-deficient AEC migration.

Exposure of WT AECs plated in 3D collagen to VEGF resulted in formation of endothelial tubes and extensive tube branching. In contrast, synectin-deficient AECs treated with VEGF developed minimal branching (Supplemental Figure 2 and Figure 3B). The exposure to GS4898 alone did not have a significant effect on branching in either WT or synectin-deficient AECs. However, GS4898 treatment in the presence of VEGF cient AECs. Interestingly, both knockdowns resulted in ERK1/2 activation, which suggests that both isoforms are involved in Aktdependent regulation of Raf1 activity (Figure 2B).

ERK1/2 activation restores arterial marker expression. One of the hallmarks of arterial morphogenic defects in synectin-null mice is a restored synectin-deficient AEC branching to the level seen in WT cells (Supplemental Figure 2 and Figure 3B).

To further assess the effect of ERK signaling on branching morphogenesis in vitro, WT and synectin-deficient AECs were plated on Matrigel in the presence or absence of GS4898 and VEGF. WT AECs 

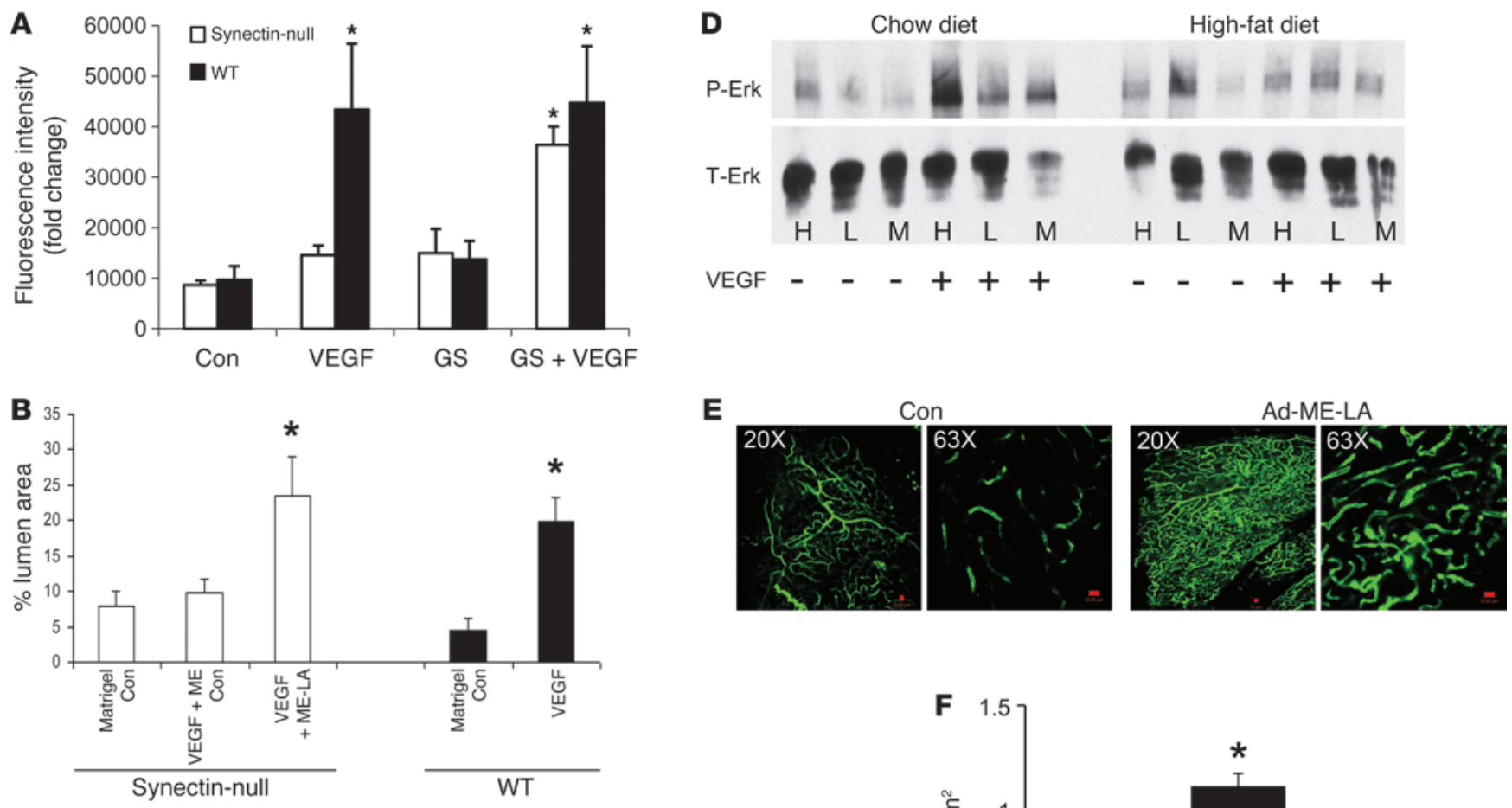

C

Chow diet

High-fat diet
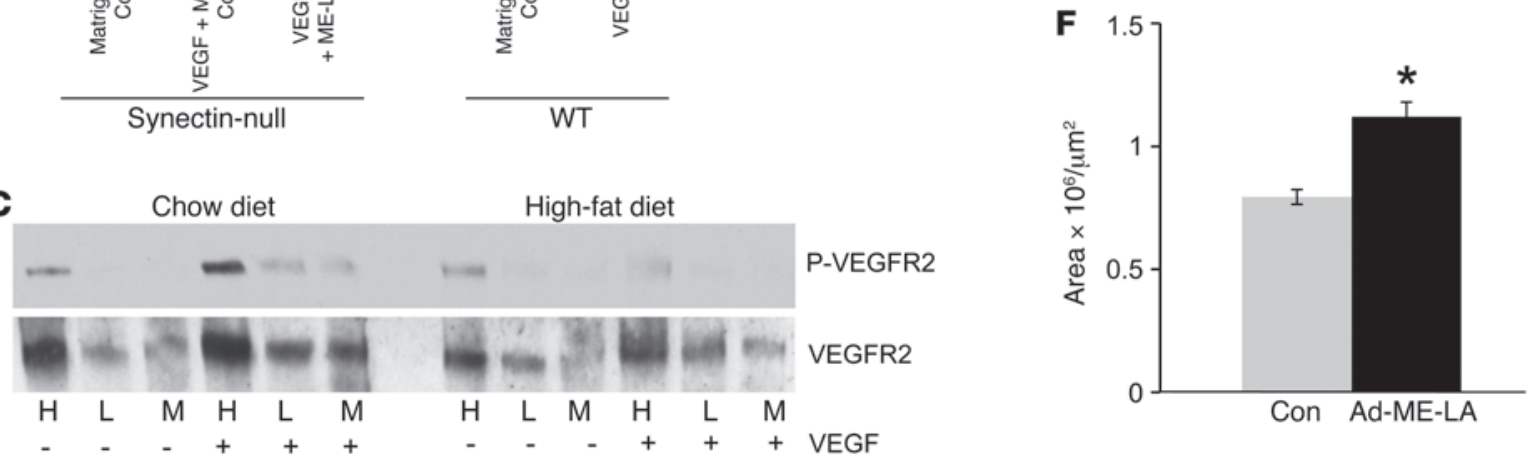

Figure 5

Restoration of ERK1/2 activation in vivo improves angiogenesis. (A) DIVAA tubes containing vehicle control or $100 \mathrm{ng} / \mathrm{ml}$ VEGF-A with heparin were placed into synectin-null $(n=8)$ or WT $(n=8)$ mice. Note restoration of neovascularization in GS4898-treated synectin-null mice in the presence of VEGF 14 days later. (B) In vivo Matrigel plug assay. Control plugs containing no virus or VEGF, or plugs containing VEGF and either Ad-ME or Ad-ME-LA virus, were implanted into synectin-null mice $(n=8)$. Matrigel plugs containing VEGF were implanted into WT mice as controls $(n=8)$. Note extensive neovascularization in VEGF plus Ad-ME-LA plugs in synectin-null mice. (C and D) VEGF-induced VEGFR2 phosphorylation in Ldlr $r^{-1}$ ApoB48-modified mice. Ldlr $r^{-1}$ ApoB48-modified mice maintained for 11 weeks on either chow $(n=6)$ or high-fat diet $(n=6)$ were injected intraperitoneally with VEGF-A (50 ng/ml), and the extent of VEGFR2 phosphorylation (anti-Y1175 Ab) (C) and ERK1/2 phosphorylation (D) was assessed by Western blotting of heart (H), liver (L), and skeletal muscle (M) tissue samples. Note decreased activation of both VEGFR2 $Y^{1175}$ and ERK1/2 in Ldlr ${ }^{-1}$ ApoB48-modified mice fed high-fat diet. (E and F) Adventitial angiogenesis assay. Ad-null control ( $n=6$ ) or Ad-ME-LA $(n=6)$ viruses in pluronic gel were added to the adventitial surface of aortas of Ldlr $r^{-1}$ ApoB48-modified mice fed a high-fat diet. Note the marked increase in neovascularization of aortas treated with Ad-ME-LA virus 21 days later. Scale bars: $10 \mu \mathrm{m}$. ${ }^{\star} P<0.05$ vs. control.

rapidly formed branching enclosures in response to VEGF (Figure 3 , C and D) while formation of such branching structures by synectin-deficient AECs was markedly impaired in the presence of VEGF alone. The addition of GS4898 to VEGF treatment fully restored branching to control levels (Figure 3, C and D). However, exposure to U0126 fully negated the effect of GS4898 supporting the notion that MEK-ERK signaling is required for branching angiogenesis.

To avoid potential nonspecific effects of chemical PI3K inhibitors, we next used constitutively active ERK constructs to study the role of ERK activation in VEGF signaling in synectin-deficient AECs. Two constructs were used: a MEK-ERK hybrid without (ME) and with a nuclear localization sequence (ME-LA). Adenoviral (Ad) transduction of synectin-deficient AECs with an empty vector (Ad-null) or the ME construct (Ad-ME) had no effect on the ability of cells to respond to VEGF treatment by branch formation on Matrigel; however, Ad-MELA fully restored branching to the level seen in WT AECs (Figure 4, A and B). Similarly, transduction with Ad-ME-LA, but not Ad-null, restored synectin-deficient AEC migration in response to VEGF to levels observed in control cells (Figure 4C).

ERK1/2 activation stimulates arteriogenesis in vivo. We next tested whether ERK1/2 activation affects vascular formation in vivo in mice. Implantation of a VEGF-containing directed in vivo angiogenesis assay (DIVAA) pellets (see Methods) into WT mice resulted in extensive vessel invasion of the pellet, with a nearly 4.5 -fold increase in pellet vascularization, which was not seen in VEGFcontaining pellets implanted into synectin-null mice. However, 

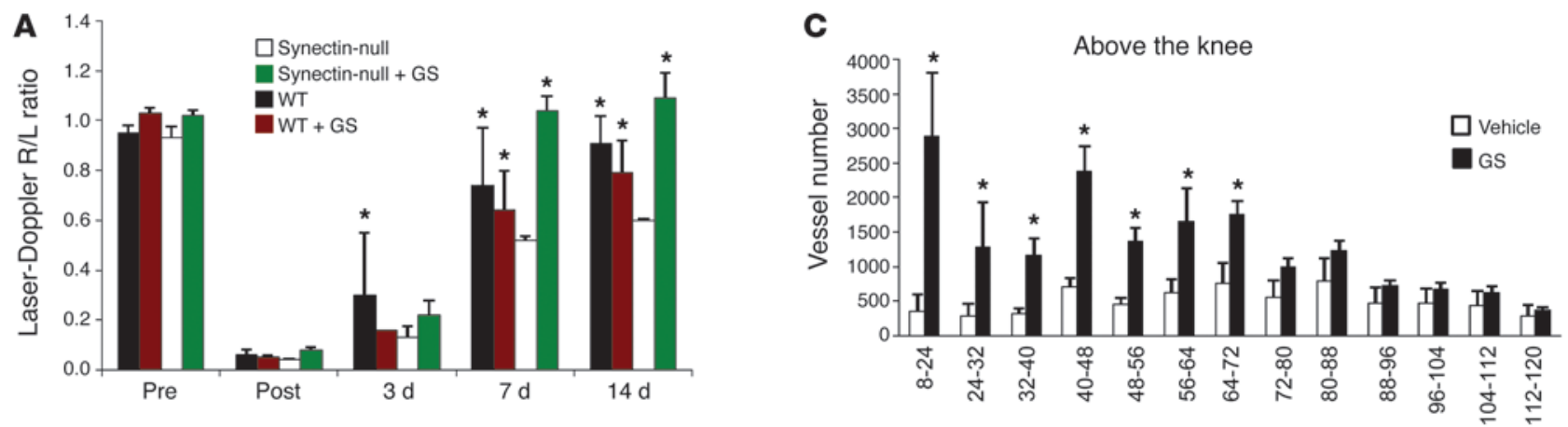

B

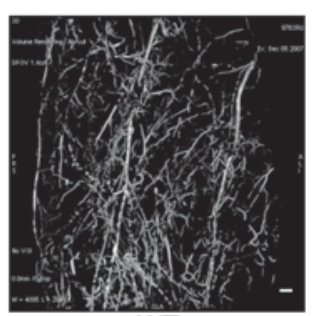

WT

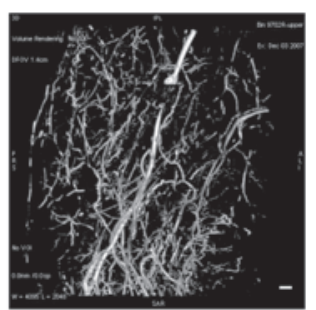

WT

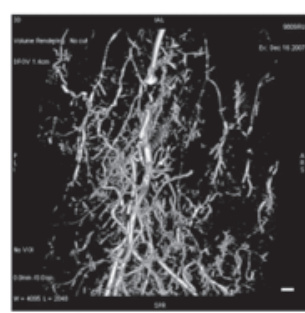

Synectin-null

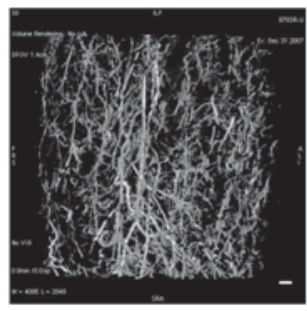

Synectin-null

D

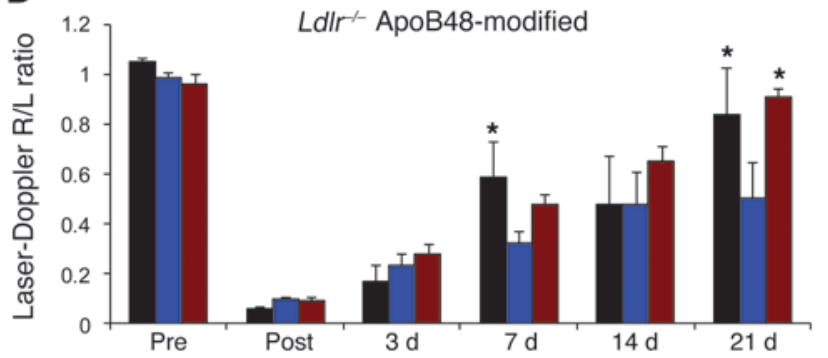

E

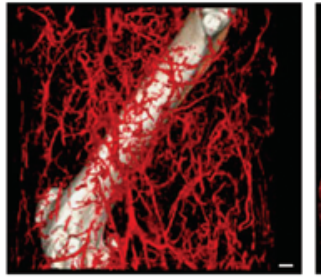

Con

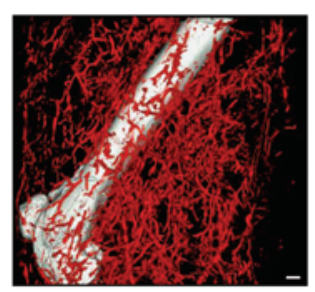

GS

Figure 6

Restoration of ERK1/2 activation in vivo improves arteriogenesis. (A-C) Common femoral artery ligation was carried out in synectin-null $(n=6)$ and WT $(n=6)$ mice as described previously (15). GS4898 was administered via the Alzet minipump. (A) Laser-Doppler analysis of blood flow recovery in the right foot, expressed as a ratio of blood flow in right to left foot (R/L). Synectin-null and WT mice received vehicle or GS4898. ${ }^{\star} P<0.05$, WT vs. synectin-null. (B) Representative $\mu$ CT carried out 14 days after the arterial ligation. (C) Quantitative analysis of the $\mu$ CT data in synectin-null mice. ${ }^{*} P<0.05$ vs. vehicle. ( $\mathbf{D}$ and $\mathbf{E}$ ) Common femoral artery ligation was performed in 12 Ldl $r^{-1-}$ ApoB48-modified mice subjected to 11 weeks of high-fat diet. One group was exposed to GS4898 administered via Alzet minipumps (red bars; $n=6$ ), while the others received vehicle (blue bars; $n=6$ ). An additional group of age-matched $L d l r^{-1}$ ApoB48-modified mice was maintained on regular chow (black bars; $n=6$ ). (D) Laser-Doppler analysis of blood flow, presented as flow ratios in right to left hindlimb. ${ }^{\star} P<0.05$ vs. vehicle. (E) Representative $\mu C T$ images. Note a major increase in the arterial development in GS4898-treated mice. Scale bars: $540 \mu \mathrm{m}$.

intraperitoneal administration of GS4898 to synectin-null mice at the time of pellet implantation restored the extent of blood vessel invasion into VEGF-containing pellets to the level seen in WT mice (Figure 5A). Similarly, the addition of an Ad-ME-LA virus, but not an Ad-ME virus, to VEGF-containing Matrigel plugs implanted in synectin-null mice restored vascularization of these plugs to the level seen in WT mice (Figure 5B).

To study the effect of ERK1/2 activation on arterial neovascularization in a different model, we first evaluated the extent of VEGF-induced VEGFR2 and ERK1/2 activation in Ldlr-/- ApoB48modified mice maintained for 12 weeks on a hyperlipidemic diet. The diet chosen causes a metabolic syndrome-like state characterized by obesity, hypertriglyceridemia, and insulin resistance (28) and, as we have previously demonstrated, extensive atherosclerotic plaque development and impaired arteriogenesis (2). After a systemic VEGF injection, the extent of VEGFR2 activation was markedly reduced in mice fed the high-fat diet, as was ERK1/2 activation (Figure 5, C and D). To test the effect of ERK1/2 activation on arteriogenesis in this model, an Ad-ME-LA or control virus was placed on the adventitial surface of the descending aorta of high-fat diet-fed $L d l r^{-/}$ApoB48-modified mice. After 3 weeks, the extent of adventitial vasculature formation was assessed using confocal microscopy, as previously described (29). Exposure to the dominant-active MEK-ERK construct resulted in a highly significant increase in the arterial vasculature (Figure 5, E and F), demonstrating the ability of ERK1/2 activation to drive arteriogenesis in a resistant mouse model.

To test further the ability of PI3K inhibition to restore impaired arteriogenesis in synectin-null and $\mathrm{Ldlr}^{-/}$ApoB48-modified mice, we used a hindlimb ischemia model. First, synectin-null animals were exposed to GS4898 or vehicle by intraperitoneal administration immediately after induction of hindlimb ischemia. In agreement with previous results (15), synectin-null mice demonstrated impaired restoration of the ipsilateral foot blood flow, as assessed by 

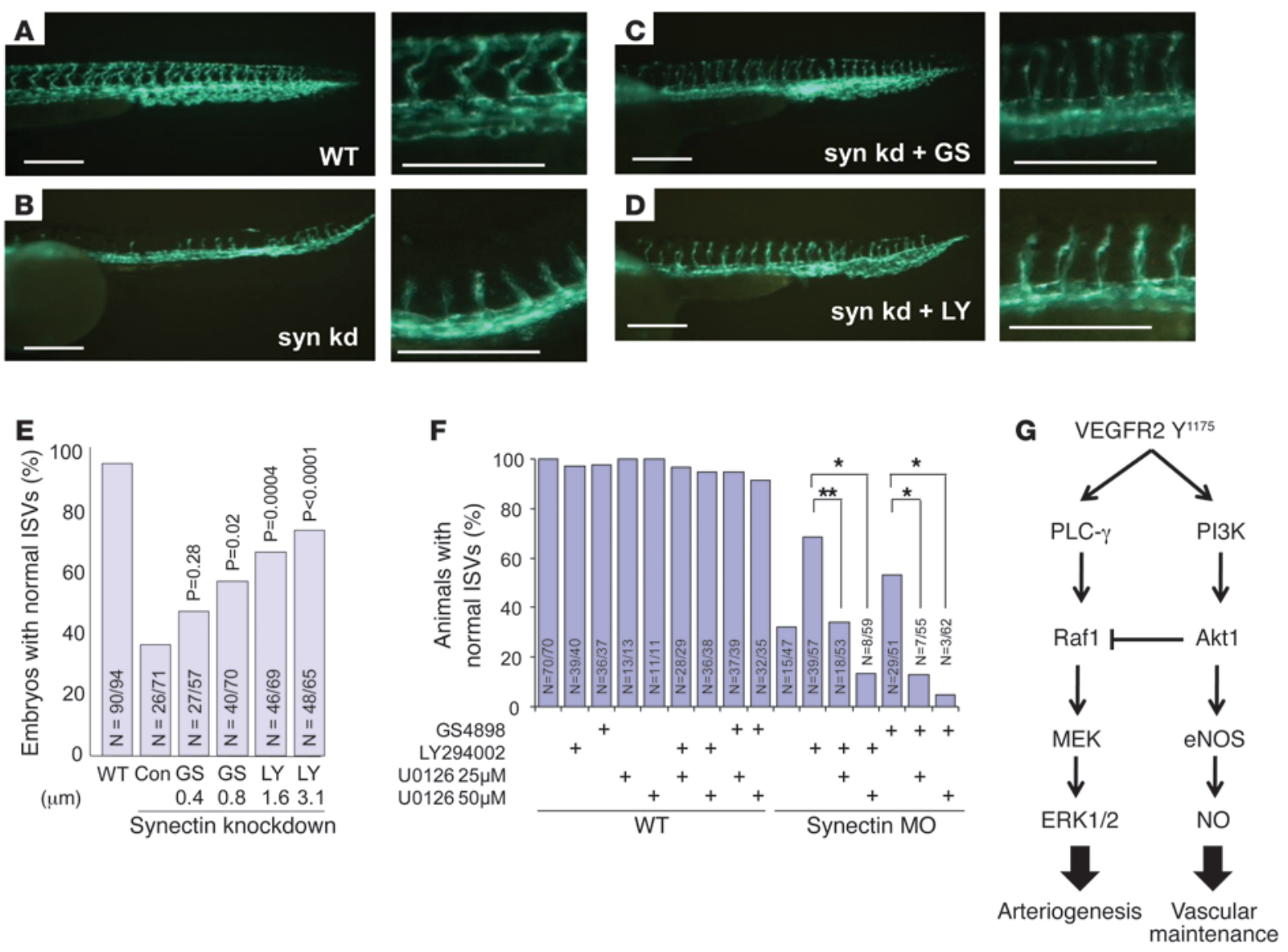

\section{Figure 7}

Restoration of ERK1/2 activation in vivo restores arteriogenesis in zebrafish. (A-E) Low- and high-power images of developing arterial vasculature in zebrafish embryos treated with vehicle (WT; A), synectin MO (B), or synectin MO plus GS4898 (C) or LY294002 (D), as described in Methods. Scale bars: $300 \mu \mathrm{m}$. (E) Quantitative analysis of ISV formation. $P$ values vs. untreated control synectin knockdown are shown. (F) Effect of MEK inhibition on PI3K downregulation-induced ISV formation. Zebrafish embryos treated with vehicle or synectin MO were exposed to GS4898, LY294002, or 2 concentrations of U0126 as indicated. No effect on the extent of ISV formation was observed in WT embryos, whereas synectin MO-treated embryos exhibited complete inhibition of ISV formation. ${ }^{*} P<0.0001$; ${ }^{\star \star} P=0.0005$. (G) Schema of PI3K/Akty1- Raf1/ER1/2 crosstalk. See text for details.

laser-Doppler perfusion imaging (Supplemental Figure 3). GS4898 treatment significantly improved perfusion as early as day 7 , with perfusion returning to the normal preligation level, whereas GS4898 treatment of control mice had little effect (Figure 6A). Such a dramatic restoration of flow implies an increase in arterial growth. This was confirmed by $\mu \mathrm{CT}$ imaging that demonstrated increased arterial morphogenesis and branching in hindlimbs of GS4898-treated compared with untreated control synectin-null mice (Figure 6B). Quantitative analysis of $\mu \mathrm{CT}$ images demonstrated a significant increase in the number of smaller $(24-72 \mu \mathrm{m})$ arteries in both the ischemic (below the knee) and nonischemic (above the knee) portions of the hindlimb (Figure 6C).

We then tested the ability of PI3K activity downregulation to stimulate arteriogenesis in $\mathrm{Ldlr}^{-1}$ ApoB48-modified mice. In agreement with previous results, 11 weeks of high-fat diet feeding led to a marked reduction in flow recovery that was fully restored by treatment with GS4898 (Figure 6D). $\mu$ CT imaging confirmed extensive arterial morphogenesis in GS4898-treated mice compared with control mice (Figure 6E).
ERK1/2 restores arterial vascular development in synectin-knockdown zebrafish embryos. Given the suppressed ERK1/2 activation and abnormal arterial morphogenesis in synectin-knockdown zebrafish embryos, we used downtitration of PI3K to evaluate the effect of ERK1/2 on arterial morphogenesis. During this process in zebrafish, intersegmental vessel (ISV) sprouting led to formation of dorsal longitudinal anastomotic vessels (DLAVs; Figure 7A). Injection of zebrafish embryos with a synectin morpholino oligonucleotide (MO) reduced ISV branching to $37 \%$ of controls and impaired DLAV formation (Figure 7, B and E), consistent with prior observations (15). Exposure of the synectin MO-injected embryos to progressively higher dosages of GS4898 or LY294002 resulted in a marked increase in ISV branching and restoration of DLAV formation (Figure $7, \mathrm{C}-\mathrm{E})$. To confirm that this effect was in fact dependent on restoration of ERK activity, WT and synectin MO-injected embryos were treated with GS4898 or LY294002 in the presence or absence of U0126. Inhibition of ERK activation with U0126 fully blocked GS4898- and LY294002-mediated restoration of ISV branching in synectin-knockdown embryos (Figure 7F). 


\section{Discussion}

The principal finding of this study is that ERK1/2 activation, by partial inhibition of PI3K activity or by introduction of a nucleartargeted constitutively active MEK-ERK construct, restored arterial morphogenesis and branching in mice and zebrafish with disrupted expression of synectin and in $\mathrm{Ldlr}^{-/}$ApoB48-modified mice fed a high-fat diet. Furthermore, we found that Akt1 was the key enzyme involved in regulation of arteriogenic response. This establishes ERK signaling as a central event in arterial morphogenesis and emphasizes a pivotal role played by the Akt1-MAPK signaling balance in this process.

Synectin deficiency is characterized by impaired arterial morphogenesis in mice, manifested as reduced numbers of arteries smaller than $70 \mu \mathrm{m}$ in diameter and markedly decreased arterial branching. Furthermore, formation of collateral arteries in adult mice after induction of ischemia is also greatly reduced. Synectin knockdown in zebrafish leads to abnormal development of ISVs or defective formation of DLAVs. In both species, the defect is limited to the arterial circulation. Although the molecular nature of the process responsible for defective arteriogenesis in synectinnull mice is not yet known, synectin-deficient AECs demonstrate decreased responsiveness to VEGF- $\mathrm{A}_{165}$ stimulation.

Similarly, advanced atherosclerosis is characterized by reduced VEGF-A responsiveness, as documented in patient samples (1) and preclinical studies $(30,31)$. In particular, $\mathrm{Ldlr}^{-/-}$ApoB48modified mice have significantly reduced arteriogenesis after common femoral artery ligation and respond poorly to growth factor administration (2).

Early on, many studies suggested that VEGF-A plays a crucial role in the establishment of arterial identity. Mice possessing only the VEGF-A $\mathrm{A}_{188}$ isoform demonstrated reduced expression of an arterial marker ephrin B2, but normal numbers of veins and capillaries in the retina (32). In zebrafish studies, injections of antisense MOs to vegf result in a severe reduction of efnb2a expression by 26 hours after fertilization, whereas flt 4 expression, a marker of venous identity, persists in ductus arteriosus. Injections of VEGF-A 121 or VEGF-A ${ }_{165}$ mRNAs result in ectopic efnb2 expression (33). VEGF can also induce expression of other arterial markers, such as notch1 and dll4, in human ECs in vitro (34). Arteries, but not veins, specifically align with peripheral nerves and follow their branching pattern in the developing mouse limb skin. This is caused by secretion of $\mathrm{VEGF}_{164}$ from sensory nerve fibers and/or Schwann cells. Lack of peripheral nerves results in defective arteriogenesis, including abnormal branching patterns and reduced expression of arterial markers, particularly reduced in small diameter vessels $(5-15 \mu \mathrm{m}$; ref. 35), a pattern consistent with abnormal arterial morphogenesis observed in synectin-null mice.

Although the data emerging from these and other studies have suggested that VEGF induces arterial specification by activating Notch signaling, perhaps via FOXC1/C2 transcription factors (36), molecular details of VEGF-induced signaling events regulating this pathway had not been established. In the endothelium, VEGF-A signals primarily via 2 high-affinity tyrosine kinase receptors, VEGFR1 (Flt-1) and VEGFR2 (Flk-1), and via neuropilin-1 (37).

Flk-1 signaling is indispensable to VEGF-induced angiogenesis and arterial specification. The key element appears to be phosphorylation of the $\mathrm{Y}^{1175}$ site that is needed for activation of both PLC $\gamma$-ERK1/2 and PI3K-Akt pathways. The importance of this site is emphasized by a study demonstrating that a knockin of Flk-1 $\mathrm{Y}^{1175} \mathrm{~F}$ mutant results in early embryonic lethality and vas- cular defects similar to those observed in a global Flk-1 knockout (38). The importance of the PLC $\gamma$-EPK $1 / 2$ pathway in mediating VEGF-driven arterial specification is also emphasized by severe and specific failure of arterial, but not venous, morphogenesis as well as absent expression of arterial markers such as efn $b 2 a$ and notch5 in zebrafish embryos after plcg1 knockdown $(39,40)$. The observed phenotype is similar to the effect of vegf knockdown and is not rescued by VEGF injections (39). Further evidence of a potential role for this pathway in arterial specification comes from observation of increased P-ERK expression in newly forming arterial vessels $(8,41)$ and from a study of gridlock mutants $(14)$. However, to our knowledge, no study to date has shown that ERK activation can promote arterial morphogenesis and branching during development and in adult tissues.

The PI3K-Akt signaling axis has also been implicated in arterial morphogenesis, although its precise role has been hard to define. On the one hand, studies in zebrafish have suggested that Akt inhibits arterial development, since mosaic expression of a constitutively active Akt1 (myr-Akt) in 1-2 cell-stage embryos forces cells expressing the construct to a predominantly venous fate, whereas expression of a dominant-negative Akt construct leads to a predominantly arterial fate (14). Similarly, increased endothelial Akt activity in Vegfr $1^{+/-}$mice resulted in impaired neovascularization in response to ischemia, while this impairment was ameliorated in Vegfr $1^{+/-} A k t 1^{+/-}$mice. Disruption of Akt 1 expression in mice has been further reported to result in enhanced angiogenesis in tumors (42).

On the other hand, in cultured ECs, Akt has been reported to activate expression of Notch1 and its ligand Dll4, both members of the arterial specification pathway (34), and a suppressed neovascularization response was observed following a different Akt 1 disruption in mice (43). The reason for these differences might stem from the changes in the balance between Akt and ERK signaling in response to different stimuli and by contribution of thrombospondin-2, a profound inhibitor of angiogenesis (44), to suppression of neovascularization. With regard to the latter, it is interesting to note that the Akt1 knockout reported by Ackah et al. (43), but not by Chen et al. (42), has profoundly increased Tsp-2 levels. The former possibility is suggested by above-cited studies demonstrating proarteriogenic effect of PI3K inhibition, and by a recent observation that the PI3K inhibitor LY294002 is able to promote vascular branching in implanted tumors (45). The likely mechanism is the observation that Akt activation results in inhibition of Raf1 activation that leads to impaired ERK1/2 activation (25).

Our findings in the present study are consistent with the concept that partial inhibition of PI3K signaling activates the MAPK pathway by suppressing Akt 1 activity and promotes arteriogenesis (Figure 7G). The PI3K inhibitors GS4898 and LY294002 restored the ability of synectin-deficient AECs to respond to VEGF-A in terms of migration and vascular structure assembly. Furthermore, PI3K inhibition in vivo improved both angiogenesis and arteriogenesis in synectin-null mice and restored ISV and DLAV formation in zebrafish. The effect was due to activation of ERK1/2, since treatment with a MEK inhibitor fully blocked all effects of partial PI3K inhibition in both mice and zebrafish. In addition, direct introduction of a constitutively active, nuclear-targeted MEK-ERK fusion construct also restored VEGF responsiveness of synectin-deficient AECs and synectin-null mice.

Importantly, this appears to be a general signaling pathway regulating arteriogenesis, as activation of ERK1/2 led to restoration of arteriogenesis in high-fat diet-fed $\mathrm{Ldlr}^{-1-}$ ApoB48-modified 
mice, a model notoriously resistant to any growth factor stimulation. The effect was mediated by Akt 1 , since suppression of Akt 1 - but not Akt2 - in synectin-deficient AECs in vitro resulted in reactivation of Raf1-MAPK signaling.

Taken together, these data strongly argue that ERK1/2 activation is necessary for effective growth of arterial vessels, both during embryogenesis and in adult tissues, and that the balance between PI3K-Akt1 and PLC $\gamma$-MAPK-VEGFR2 signaling pathways is one of the key factors in regulations of arterial versus venous fate mapping and growth of arterial vasculature. Interventions aimed at activation of ERK1/2 signaling, either by direct activation or by suppression of Akt1-mediated inhibition, may prove effective in stimulating arterial growth in patients with atherosclerotic vascular disease.

\section{Methods}

Study approval. All animal studies were approved by the Institutional Animal Care and Use Committees of Dartmouth College and Yale University.

Isolation and culture of primary AECs and antibodies. AECs were isolated and passaged from synectin-null or WT mice as previously described (15). Antibodies for Western blotting were from the following sources, unless otherwise indicated: P-eNOS (Cell Signaling), eNOS (Zymed), S6K (Epitomics), P-GSK3 $\beta$, GSK3 $\beta$, P-4EBP1, 4EBPI, P-ERK1/2, and ERK1/2 (all from Cell Signaling).

Expression of constitutively active ERK2. Constitutive active ME and ME-LA constructs (gift of M.H. Cobb, University of Texas Southwestern Medical Center, Dallas; ref. 46) were shuttled into Ad vectors using the ViraPower Adenovirus Expression System (Invitrogen) as described by the manufacturer. Briefly, the constructs were subcloned into the pENTR (Invitrogen), and then cDNA inserts were transferred into $\mathrm{pAd} / \mathrm{CMV} / \mathrm{V} 5$-DEST vector using the Gateway system and LR Clonase (Invitrogen). The Ads were then produced using HEK 293A cells.

Infection of AECs with generated Ad coding was performed by adding $100 \mu \mathrm{l}$ of the recombinant virus supernatant in AEC culture media at a titer of $10^{8} \mathrm{PFU}$, using Ad-GFP as a control. Infection efficiency was determined by fluorescent microscopy for GFP and confirmed by anti-ERK2 staining. Typically, more than $90 \%$ of cells were successfully transduced. Transduced AECs were cultured for an additional 48 hours before being used for migration in in vitro Matrigel or in vivo Matrigel plug assay.

Quantitative PCR. RNA was prepared using a Qiagen RNaeasy kit following the instructions from the manufacturer. cDNA was synthesized using iScript cDNA Synthesis kit (BioRad). Gene expression was then analyzed by quantitative PCR using a BioRad SYBR kit on a BioRad CFX96 system. Primers used for quantitative PCR were all purchased from SA Biosciences Corp. All gene expressions were normalized to Gapdh mRNA levels. Primer sequences were as follows: EphrinB2 forward, 5 '-CTCAACTGTGCCAGACCAGA-3'; EphrinB2 reverse, 5'-CTTGTTGGACCGTGATTCCT-3'; Hey2 forward, 5'-GTTCCGCTAGGCGACAGTAG-3'; Hey2 reverse, 5'-GTTGTCGGTGAATTGGACCT-3'; Gapdh forward, 5'-AACTTTGGCATTGTGGAAGG-3'; Gapdh reverse, 5'-ACACATTGGGGGTAGGAACA-3'; Dll4 forward, 5'-ACCTTTGGCAATGTCTCCAC-3'; Dll4 reverse, 5'-TTGGATGATGATTTGGCTGA-3'.

siRNA expression knockdown. Mouse $\mathrm{p} 110 \alpha$ (catalog no. GS18706) and p110 $\beta$ (catalog no. GS74769) Flexitube siRNAs and AllStars Negative Control siRNA (catalog no. SI03650318) were purchased from Qiagen. Cells cultured in 6-well plates at 50\% confluence were transfected with $5 \mathrm{nmol}$ siRNA using TransPass R2 Transfection Reagent (NEB) following the manufacturer's instructions. 48 hours after siRNA transfection, the cells were used for experiments. The knockdown efficiency was determined by quantitative PCR or Western blotting.
Cell migration. For the modified Boyden chamber migration assays, the chambers were placed in 24-well dishes filled with DMEM containing $0.5 \%$ FBS. $4 \mu \mathrm{M}$ GS4898, 25 ng/ml VEGF-A, or $10 \mu \mathrm{M}$ U0126 (R\&D Systems) was added to the media as required. AECs were plated in the upper compartment. 24 hours later, the filters were removed and submerged in $4 \%$ paraformaldehyde (Electron Microscopy Sciences) for 30-40 minutes at room temperature, then labeled with Alexa Fluor 568 Phalloidin (1:200 dilution; Invitrogen) for 30 minutes at $37^{\circ} \mathrm{C}$. The dye was discarded, the cells were washed twice, and the membranes were then cut and placed on a microscopy slide. Images were obtained using a confocal fluorescence microscope and analyzed using NIH Scion/Image J Imaging Analysis.

$3 D$ collagen tube branching assay. AECs from synectin-null or WT mice were plated in 3D collagen-1 matrix and allowed to form branching tubular structures as previously described (15). 24 hours later, the medium was discarded, and 3D collagen matrix was washed twice with PBS, fixed with $4 \%$ paraformaldehyde for 30-40 minutes at room temperature, labeled with Alexa Fluor 568 Phalloidin (1:200 dilution; Invitrogen) for 30 minutes at $37^{\circ} \mathrm{C}$, and then covered with ProLong Gold antifade reagent (P36954; Invitrogen). Images were collected using a LSM 510 META confocal fluorescence microscope. The extent of branching was determined using NIH Scion/Image J Imaging software as previously described (15).

In vitro Matrigel assay. For the in vitro Matrigel assay, $2 \times 10^{4}$ synectindeficient or WT AECs were plated on 12-well culture plates coated with growth factor reduced Matrigel (200 $\mu \mathrm{l} /$ well; BD). VEGF-A $(100 \mathrm{ng} / \mathrm{ml})$ and/or GS4898 was also added to the wells at this time. After 16-24 hours, the formed cellular networks were stained with Alexa Fluor 568 Phalloidin (1:200 dilution; Invitrogen) and visualized by fluorescence confocal microscope. The total tube length and enclosure area were quantified using NIH Image J software in 6-8 randomly chosen fields.

DIVAA. The DIVAA assay was performed as described previously (47), with the following modifications. DIVAA angioreactors lumens (Trevigen) were filled with high-concentration basement membrane extract containing recombinant VEGF $(100 \mathrm{ng} / \mathrm{ml})$ with heparin. The angioreactors were inverted and incubated at $37^{\circ} \mathrm{C}$ for 1 hour to allow gel formation and then implanted subcutaneously into flanks of synectin-null and WT mice under anesthesia. For FITC-dextran assay, 14 days later the mice were injected intravenously with FITC-dextran ( $25 \mathrm{mg} / \mathrm{ml}, 100 \mu \mathrm{l} /$ mouse; Sigma-Aldrich). 20 minutes later, the angioreactors were removed from the skin pockets and photographed with a dissecting microscope. CultrexCultrex Basement Membrane Extract/ vessel complex was taken out of angioreactors, put into sterile microtubes, and incubated for $1-3$ hours at $37^{\circ} \mathrm{C}$. Then the samples were centrifuged at $15,000 \mathrm{~g}$ for 5 minutes at room temperature, and the supernatant was placed in 96-well plate for fluorescence analysis. For FITC-lectin assay, the basement membrane extract/vessel complex was directly taken out of angioreactors and processed according to the manufacturer's protocol, and then the pellets were used for fluorescence analysis. The fluorescence intensity was measured at a wavelength of excitation $485 \mathrm{~nm}$ and emission $510 \mathrm{~nm}$ with BioTek MultiDetection Microplate Reader Synergy HT (BioTek Instruments Inc.)

Matrigel plug in vivo angiogenesis assay. The Matrigel plug assay was performed by injecting WT and synectin-null male mice intradermally with $0.5 \mathrm{ml}$ Matrigel premixed with VEGF-A $(100 \mathrm{ng} / \mathrm{ml})$ and either the Ad-ME vector or the Ad-ME-LA vector $\left(10^{8} \mathrm{PFU} / \mathrm{ml}\right)$ under anesthesia. The mice were sacrificed 11 days after injection, and Matrigel plugs were removed and fixed with $4 \%$ paraformaldehyde overnight at $4{ }^{\circ} \mathrm{C}$ and then in $10 \%$ formaldehyde before embedding in paraffin. The paraffin blocks were cut into 5 - $\mu \mathrm{m}$-thick sections and stained with hematoxylin and eosin. Randomly chosen sections were analyzed by confocal microscopy using NIH Image J software.

Hindlimb ischemia model. WT or synectin-null mice (8-10 weeks old) or Ldlr ${ }^{-1}$ ApoB48-modified mice (24 weeks old) maintained for 11 weeks on a modified Paigen diet as described previously (29) were used for all studies. 
Mice were subjected to common femoral artery ligation as described previously (15). Serial assessment of blood flow in the foot was carried out using the Moor LDI system with Moor LDI measurement V3.09 software (Moor Instruments) as previously described (15). ALZET osmotic minipumps (model 1002, $0.25 \mu \mathrm{l} / \mathrm{h}, 14$-day delivery; ALZET) containing either saline and DMSO or GS4898 were implanted intraperitoneally under anesthesia 1 day prior to femoral artery ligation.

$\mu C T$ analysis. $\mu$ CT studies were carried out as described previously $(2,48)$. The ligated right hindlimb vasculature was scanned with a high-resolution $\mu \mathrm{CT}$ imaging system (GE eXplore Locus SP; GE Healthcare) set to an $8-\mu \mathrm{m}$ effective detector pixel size. $\mu \mathrm{CT}$ operated at $80-\mathrm{kVp}$ X-ray tube voltage, 80- $\mu$ A tube current, $3,000 \mathrm{~ms}$ per frame, $1 \times 1$ detector binning model, 720 views, and $0.5^{\circ}$ increments per view to acquire a set of contiguous axial VFFformatted images through each hindlimb. With the use of Microview Software (GE Healthcare), the raw data were corrected and reconstructed with voxels of dimensions $24 \mu \mathrm{m} \times 24 \mu \mathrm{m} \times 24 \mu \mathrm{m}$ to visualize the whole vascular tree. For quantification of the vascular tree, both calf and thigh portions were individually selected with voxels of dimensions $16 \mu \mathrm{m} \times 16 \mu \mathrm{m} \times 16 \mu \mathrm{m}$, up to 1,250 frames per rotation for reconstruction with a cone-beam algorithm. The reconstructed $\mu \mathrm{CT}$ data were transferred to a Dell Dimension Workstation with real-time 3D volume rendering software (version 3.1; Vital Images Inc.) and Microview software (version 1.15; GE Healthcare). Modified Image J software and Image Pro Plus (Media Cybernetics) were used to analyze the total area and volume. Data are expressed as vascular segment number, representing total number of vessels, of specified diameter, counted in 250 reformatted cross-sections for thigh or 250 sections for calf in 3D.

Stimulation of adventitial angiogenesis. Female $\mathrm{Ldlr}{ }^{--}$ApoB48-modified mice (12 weeks old) were fed modified Paigen diet for 12 weeks. Next, the mice were anesthetized, intubated, and taped down to a disinfected operating field. An oblique $8-\mathrm{mm}$ incision of the skin was made about $6 \mathrm{~mm}$ from the left sternal border toward the left armpit. A blunt dissection was used to separate both layers of thoracic muscle. The chest was opened at the sixth intercostal space, and a retractor was inserted and spread about 4-5 $\mathrm{mm}$ to expose the descending aorta. Ad-ME-LA (20 IU) or Ad-null in 40\% pluronic gel was added directly onto the descending aorta with a needle. The chest was closed by suturing open ribs, the muscle layer, and skin. The mice were maintained on the same diet for an additional 21 days after surgery. At that point, mice were sacrificed and perfused with fluorescein-labeled Lycopersicon esculentum lectin (tomato; Vector Labs), and descending aorta whole mounts were imaged by confocal microscopy as previously described (29).

Analysis of vessel density. Descending aortas were removed with a wide margin and made permeable in an overnight incubation at $4^{\circ} \mathrm{C}$ in PBS containing $1 \%$ BSA and $0.1 \%$ Triton X. Fluorescein-labeled, L. esculentum lectin-perfused adventitial vessels were imaged on a Zeiss LSM-510 META point scanning confocal microscope, and $z$ stacks were collected $(\times 10$ and $\times 20$ objectives, 0.7 scan zoom, $135 \mu \mathrm{m}$ pinhole, and $325.8 \times 325.8 \times 9.0 \mu \mathrm{m}$ stack size). The $z$ stack vessel images were reconstructed using Volocity version 3.7 software (Improvision).

In vivo VEGF responsiveness. Male Ldlr $/$ ApoB48-modified mice $(n=12)$ were fed normal chow diet for 12 weeks, at which time 6 mice began Paigen diet without cholate for 11 weeks and the remaining 6 mice continued the chow diet. Following the period of atherogenic diet, 3 mice from each diet group received an intraperitoneal injection of VEGF-A $(50 \mathrm{ng} / \mathrm{ml}$ saline), and 3 mice per group received $1 \mathrm{ml}$ saline. The mice were sacrificed 4 hours after receiving the respective injections. Various organs (heart, liver, and skeletal muscle) were immediately removed from each mouse and homogenized in a lysis buffer containing protease inhibitors. Protein concentrations from all samples were determined by Bradford assay, and equivalent amounts of protein were gel-resolved and transferred to nitrocellulose membranes. Membranes were probed for total ERK1/2, P-ERK1/2, total VEGFR2, and P-VEGFR2 Y1175 (all antibodies from Cell Signaling). The binding reactions were amplified with HRP-conjugated secondary antibodies and detected with either SuperSignal West Pico or Femto chemiluminescent substrate (Thermo Scientific). Studies in synectin-null mice $(n=6)$ were carried out in a similar manner, except that mice were maintained on the regular chow diet.

Zebrafish Western analysis. 1 cell stage embryos were injected with $12 \mathrm{ng}$ synectin MO. At approximately 8-ss, embryos were treated with 20, 50, or $100 \mu \mathrm{M}$ GS4898 or with DMSO as a control. Embryos were homogenized in SDS lysis buffer at 18- to 20-ss. Lysates from an equivalent number of embryos (approximately 3 ) were loaded on $10 \%$ SDS polyacrylamide gel and transferred to a PVDF membrane. Membranes were blocked with 5\% nonfat milk solution and probed with P-p44/42 MAPK ( $\left.\mathrm{T}^{202} / \mathrm{T}^{204}\right) \mathrm{Ab}$ or $\mathrm{p} 44 / 42 \mathrm{MAPK} A b$ (Cell Signaling). After washes, membranes were incubated with goat anti-rabbit HRP-conjugated secondary Ab (Calbiochem). Following extensive washes, membranes were developed with $\mathrm{ECL}^{+}$chemiluminescence reagents and scanned with a Typhoon imager (GE Healthcare). Band intensities were quantified using ImageJ image analysis software. Ratios between P-MAPK and total MAPK were calculated.

Zebrafish synectin rescue assay. 1-cell stage fli1-eGFP transgenic zebrafish embryos were injected with $12 \mathrm{ng}$ synectin MO. At approximately 9-ss, embryos were treated with $0.4,0.8$, or $1.6 \mu \mathrm{M}$ GS4898; with 1.6 or $3.2 \mu \mathrm{M}$ LY294002; or with DMSO as a control. Compounds were washed off after 4 hours of treatment, and formation of ISVs was monitored at approximately 40 hours after fertilization. For assessing the effects of U0126, MOinjected and control embryos were treated at approximately 9-ss with 25 or $50 \mu \mathrm{M}$ U0126 plus $0.8 \mu \mathrm{M}$ GS4898, 3.1 $\mu \mathrm{M}$ LY294002, or DMSO.

Statistics. All data are shown as mean \pm SEM. Statistical analysis was carried out using 2-tailed indirect Student's $t$ test, $\chi^{2}$ analysis, or 1-way ANOVA with a post-hoc least significant difference test, with or without repeated measures, with SPSS and the R 2.0.1 statistical software package as appropriate. Differences were considered significant at a $P<0.05$ level.

\section{Acknowledgments}

This work was supported in part by NIH grants HL84619 and HL53793 (to M. Simons), HL079267 (to R.T. Peterson), and HL69948 (to M.J. Mulligan-Kehoe).

Received for publication May 12, 2009, and accepted in revised form January 6, 2010.

Address correspondence to: Michael Simons, Section of Cardiovascular Medicine, Yale University School of Medicine, 333 Cedar St., P.O. Box 208017, New Haven, CT 06520-8017. Phone: 203.785.7000; Fax: 203.785.7144; E-mail: michael.simons@yale.edu.
1. Sasso FC, et al. Increased vascular endothelial growth factor expression but impaired vascular endothelial growth factor receptor signaling in the myocardium of type 2 diabetic patients with chronic coronary heart disease. J Am Coll Cardiol. 2005;46(5):827-834.

2. Tirziu D, et al. Delayed arteriogenesis in hypercholesterolemic mice. Circulation. 2005;112(16):2501-2509.

3 . Waltenberger J. Impaired collateral vessel develop- ment in diabetes: potential cellular mechanisms and therapeutic implications. Cardiovasc Res. 2001;49(3):554-560.

4. Simons M. Angiogenesis: where do we stand now? Circulation. 2005;111(12):1556-1566.

5. Urness LD, Sorensen LK, Li DY. Arteriovenous malformations in mice lacking activin receptor-like kinase-1. Nat Genet. 2000;26(3):328-331.

6. Benjamin LE, Hemo I, Keshet E. A plasticity win- dow for blood vessel remodelling is defined by pericyte coverage of the preformed endothelial network and is regulated by PDGF-B and VEGF. Development. 1998;125(9):1591-1598.

7. Paye JM, Phng LK, Lanahan AA, Gerhard H, Simons M. Synectin-dependent regulation of arterial maturation. Dev Dyn. 2009;238(3):604-610.

8 . Eitenmuller I, et al. The range of adaptation by collateral vessels after femoral artery occlusion. Circ 
Res. 2006;99(6):656-662.

9. Jalali S, et al. Shear stress activates p60src-Ras-MAPK signaling pathways in vascular endothelial cells. Arterioscler Thromb Vasc Biol. 1998;18(2):227-234.

10. Sumpio BE, et al. MAPKs (ERK1/2, p38) and AKT can be phosphorylated by shear stress independently of platelet endothelial cell adhesion molecule-1 (CD31) in vascular endothelial cells. J Biol Chem. 2005;280(12):11185-11191.

11. Lamont RE, Childs S. MAPping out arteries and veins. Sci STKE. 2006;2006(355):pe39.

12. Weinstein BM, Stemple DL, Driever W, Fishman MC. Gridlock, a localized heritable vascular patterning defect in the zebrafish. Nat Med. 1995; 1(11):1143-1147.

13. Zhong TP, Rosenberg M, Mohideen MA, Weinstein $B$, Fishman MC. gridlock, an HLH gene required for assembly of the aorta in zebrafish. Science. 2000;287(5459):1820-1824

14. Hong CC, Peterson QP, Hong JY, Peterson RT. Artery/vein specification is governed by opposing phosphatidylinositol-3 kinase and MAP kinase/ ERK signaling. Curr Biol. 2006;16(13):1366-1372.

15. Chittenden TW, et al. Selective regulation of arterial branching morphogenesis by synectin. Dev Cell. 2006;10(6):783-795.

16. Dedkov EI, et al. Synectin/syndecan-4 regulate coronary arteriolar growth during development. Dev Dyn. 2007;236(7):2004-2010.

17. Gao Y, Li M, Chen W, Simons M. Synectin, syndecan-4 cytoplasmic domain binding PDZ protein, inhibits cell migration. J Cell Physiol. 2000; 184(3):373-379.

18. Cai H, Reed RR. Cloning and characterization of neuropilin-1-interacting protein: a PSD-95/Dlg/ ZO-1 domain-containing protein that interacts with the cytoplasmic domain of neuropilin-1. J Neurosci. 1999;19(15):6519-6527.

19. Lou X, Yano H, Lee F, Chao MV, Farquhar MG. GIPC and GAIP form a complex with TrkA: a putative link between $\mathrm{G}$ protein and receptor tyrosine kinase pathways. Mol Biol Cell. 2001;12(3):615-627.

20. Blobe GC, Liu X, Fang SJ, How T, Lodish HF. A novel mechanism for regulating transforming growth factor beta (TGF-beta) signaling. Functional modulation of type III TGF-beta receptor expression through interaction with the PDZ domain protein, GIPC. J Biol Chem. 2001;276(43):39608-39617.

21. Lanahan AA, Chittenden TW, Mulvihill E, Smith K, Schwartz S, Simons M. Synectin-dependent gene expression in endothelial cells. Physiol Genomics. 2006;27(3):380-390.

22. Prahst C, et al. Neuropilin-1-VEGFR-2 complexing requires the PDZ-binding domain of neuropilin-1. J Biol Chem. 2008;283(37):25110-25114.

23. Salikhova A, et al. Vascular endothelial growth factor and semaphorin induce neuropilin-1 endocytosis via separate pathways. Circ Res. 2008;103(6):e71-e79.

24. Elfenbein A, Rhodes JM, Meller J, Schwartz MA, Matsuda M, Simons M. Suppression of RhoG activity is mediated by a syndecan 4-synectin-RhoGDI1 complex and is reversed by PKCalpha in a Rac1 activation pathway. J Cell Biol. 2009;186(1):75-83.

25. Zimmermann S, Moelling K. Phosphorylation and regulation of Raf by Akt (protein kinase B). Science. 1999;286(5445):1741-1744.

26. Graupera M, et al. Angiogenesis selectively requires the p110alpha isoform of PI3K to control endothelial cell migration. Nature. 2008; 453(7195):662-666.

27. Jia S, et al. Essential roles of PI(3)K-p110beta in cell growth, metabolism and tumorigenesis. Nature. 2008;454(7205):776-779.

28. Hartvigsen K, et al. A diet-induced hypercholesterolemic murine model to study atherogenesis without obesity and metabolic syndrome. Arterioscler Thromb Vasc Biol. 2007;27(4):878-885.

29. Drinane $M$, et al. The antiangiogenic activity of rPAI-1(23) inhibits vasa vasorum and growth of atherosclerotic plaque. Circ Res. 2009;104(3):337-345.

30. Metais C, Li J, Li J, Simons M, Sellke FW. Effects of coronary artery disease on expression and microvascular response to VEGF. Am J Physiol. 1998; 275(4 Pt 2):H1411-H1418.

31. Voisine $P$, et al. Inhibition of the cardiac angiogenic response to exogenous vascular endothelial growth factor. Surgery. 2004;136(2):407-415.

32. Stalmans I, et al. Arteriolar and venular patterning in retinas of mice selectively expressing VEGF isoforms. J Clin Invest. 2002;109(3):327-336.

33. Lawson ND, Weinstein BM. In vivo imaging of embryonic vascular development using transgenic zebrafish. Dev Biol. 2002;248(2):307-318.

34. Liu ZJ, et al. Regulation of Notch1 and Dll4 by vascular endothelial growth factor in arterial endothelial cells: implications for modulating arteriogenesis and angiogenesis. Mol Cell Biol. 2003;23(1):14-25.

35. Mukouyama YS, Shin D, Britsch S, Taniguchi M, Anderson DJ. Sensory nerves determine the pattern of arterial differentiation and blood vessel branch- ing in the skin. Cell. 2002;109(6):693-705.

36. De Val S, Black BL. Transcriptional control of endothelial cell development. Dev Cell. 2009; 16(2):180-195.

37. Olsson AK, Dimberg A, Kreuger J, Claesson-Welsh L. VEGF receptor signalling - in control of vascular function. Nat Rev Mol Cell Biol. 2006;7(5):359-371.

38. Takahashi T, Yamaguchi S, Chida K, Shibuya M. A single autophosphorylation site on KDR/Flk-1 is essential for VEGF-A-dependent activation of PLCgamma and DNA synthesis in vascular endothelial cells. EMBO J. 2001;20(11):2768-2778

39. Lawson ND, Mugford JW, Diamond BA, Weinstein BM. phospholipase C gamma- 1 is required downstream of vascular endothelial growth factor during arterial development. Genes Dev. 2003; 17(11):1346-1351.

40. Isogai S, Lawson ND, Torrealday S, Horiguchi M, Weinstein BM. Angiogenic network formation in the developing vertebrate trunk. Development. 2003;130(21):5281-5290.

41. Deindl E. Arteriogenesis: a focus on signal transduction cascades and transcription factors. Thromb Haemost. 2007;98(5):940-943.

42. Chen J, et al. Akt1 regulates pathological angiogenesis, vascular maturation and permeability in vivo. Nat Med. 2005;11(11):1188-1196.

43. Ackah E, et al. Akt1/protein kinase Balpha is critical for ischemic and VEGF-mediated angiogenesis. J Clin Invest. 2005;115(8):2119-2127.

44. Kyriakides TR, et al. Mice that lack thrombospondin 2 display connective tissue abnormalities that are associated with disordered collagen fibrillogenesis, an increased vascular density, and a bleeding diathesis. J Cell Biol. 1998;140(2):419-430.

45. Sourbier C, et al. The phosphoinositide 3-kinase/ Akt pathway: a new target in human renal cell carcinoma therapy. Cancer Res. 2006;66(10):5130-5142.

46. Robinson MJ, Stippec SA, Goldsmith E, White MA, Cobb MH. A constitutively active and nuclear form of the MAP kinase ERK2 is sufficient for neurite outgrowth and cell transformation. Curr Biol. 1998;8(21):1141-1150.

47. Bianco C, et al. Role of human cripto-1 in tumor angiogenesis. J Natl Cancer Inst. 2005;97(2):132-141.

48. Li W, et al. High-resolution quantitative computed tomography demonstrating selective enhancement of medium-size collaterals by placental growth factor- 1 in the mouse ischemic hindlimb. Circulation. 2006;113(20):2445-2453 